\title{
Classical Anomalies for Spinning Particles
}

\author{
Jorge Gamboa $^{1 *}$ and Mikhail Plyushchay ${ }^{2,3 \dagger}$ \\ ${ }^{1}$ Departamento de Fúsica, Universidad de Santiago de Chile \\ Casilla 30\%, Santiago 2, Chile \\ ${ }^{2}$ Institute for High Energy Physics, Protvino \\ Moscow Region, 142284 Russia \\ ${ }^{3}$ Departamento de Fisica, Universidade Federal de Juiz de Fora \\ 36036-330 Juiz de Fora, MG Brazil
}

\begin{abstract}
We discuss the phenomenon of classical anomaly. It is observed for $3 D$ Berezin-Marinov (BM), Barducci-Casalbuoni-Lusanna (BCL) and Cortés-PlyushchayVelázquez (CPV) pseudoclassical spin particle models. We show that quantum mechanically these different models correspond to the same $P, T$-invariant system of planar fermions, but the quantum system has global symmetries being not reproducible classically in full in any of the models. We demonstrate that the specific U(1) gauge symmetry characterized by the opposite coupling constants of spin $s=+1 / 2$ and $s=-1 / 2$ states has a natural classical analog in CPV model but can be reproduced in BM and BCL models in an obscure and rather artificial form. We also show that $\mathrm{BM}$ and BCL models quantum mechanically are equivalent in any odd-dimensional space-time, but describe different quantum systems in even space-time dimensions.
\end{abstract}

PACS numbers: 12.90.+b, 11.30.-j

Keywords: spin particle models, (super)symmetries, anomalies.

Nucl. Phys. B 512 (1998) 485-504

*e-mail: jgamboa@lauca.usach.cl

${ }^{\dagger}$ e-mail: plyushchay@mx.ihep.su 


\section{Introduction}

When global or local symmetries do not survive under quantization, i.e. some classical symmetries are lost at the quantum level, we have global [困] or local quantum anomalies [2, 3]. Since the quantization is not unique, different quantum systems corresponding to one and the same classical system can be constructed and sometimes symmetries can be restored by modifying appropriately the quantization procedure. A priori there is no obstruction for existence of the inverse picture when different classical models correspond to one and the same quantum system having symmetries to be not reproducible at least in some of these classical models.

The present paper is devoted to investigation of such a phenomenon of classical anomaly for the $P, T$-invariant $3 D$ fermion system [4, 5, 6].

The phenomenon of classical anomaly will be revealed here in the following aspect. We shall investigate classical and quantum theory of three $3 D$ spin particle models. These are the Berezin-Marinov (BM) [7, 8, 9], Barducci-Casalbuoni-Lusanna (BCL) [10 and CortésPlyushchay-Velázquez (CPV) [11, 6] pseudoclassical models. All these three models have the same number of gauge symmetries generated by the corresponding first class constraints. Dirac quantization procedure supplies us in all three cases with the mass shell condition and with the $P, T$-invariant system of $3 D$ Dirac equations which are the quantum counterparts of the corresponding classical constraints. Therefore, the quantization of these different models leads to one and the same quantum system of the $P, T$-invariant massive planar fermions. We shall get the set of integrals of motion of the quantum system (generators of global symmetries) and establish the (super)algebras formed by them. Then we shall find that

1) not all the quantum integrals of motion have classical analogs in CPV model,

2) though BCL and BM models contain all the formal classical analogs of the quantum integrals, nevertheless the corresponding symmetry (super)algebras can be reproduced classically in BCL model only partially, whereas the BM model does not allow us to reproduce even a part of corresponding (super)algebras. On the other hand, we shall see that some properties of (super)symmetry generators being not reproducible in BCL model, are reproduced classically in $\mathrm{CPV}$ model.

Therefore, we shall show that all the three models reflect classically some different parts of properties of the corresponding quantum system and neither of them reproduce the quantum symmetry properties in full.

In addition, we shall demonstrate that the so called $\sigma_{3}$ (or $\tau_{3}$ in terminology of Refs. [1]) local U(1) gauge symmetry, characterized by the opposite coupling constants of spin $s=+1 / 2$ and $s=-1 / 2$ states and denoted further as $\mathrm{U}_{\sigma_{3}}(1)$, has a natural classical analog in CPV model but can be reproduced in BM and BCL models in an obscure and rather artificial form.

Just from the described features of classical anomaly taking place for the concrete system to be investigated here it is clear how important role this phenomenon may play. Indeed, if the quantization of some model gives the necessary quantum system, it may turn out that not all the quantum symmetry properties of the system are automatically reflected at the classical level. On the other hand, it may turn out that in order to reflect classically all the quantum symmetry properties of the system, it is necessary to use different classical models taking into account different aspects of symmetry properties of the same quantum system. 
We shall discuss other possible consequences of the observed phenomenon in last Section.

The paper is organized as follows. In Section 2 we analyze the classical properties of BM and BCL models. We show that these models are closely related but not equivalent: the BCL model has one odd integral of motion which is absent from the BM model. This difference has no consequences from the point of view of quantum theory of the models in the case of odd-dimensional space-time but turns out to be crucial under quantization in even space-time dimensions, in particular, in $3+1$ dimensions where the models were constructed originally. The quantization of $3 D \mathrm{BM}$ and BCL models by the Dirac method is realized in Section 3. In Section 4 we consider the CPV model and compare it with the BM and BCL models. The peculiarity of the CPV model is that it does not admit, even local, gauge conditions [12]. In Section 5 we discuss the quantum symmetries of the corresponding $P, T$-invariant planar fermion system. Section 6 is devoted to the discussion of classical anomalies. In Section 7 we analyze the classical counterparts of the $\mathrm{U}_{\sigma_{3}}(1)$ gauge theory. Section 8 contains some concluding remarks and discussion. Appendix is devoted to the quantization of BM and BCL models in even-dimensional space-time.

\section{$2 \quad$ BM and BCL models}

\subsection{Lagrangians}

The Lagrangians of BM and BCL models, introduced originally in $3+1$ dimensions, in general case of $D$-dimensional space-time are given by

$$
\begin{gathered}
L_{B M}=\frac{1}{2 e}\left(\dot{x}_{\mu}+i \lambda \xi_{\mu}\right)^{2}-\frac{e}{2} m^{2}-i m \lambda \xi_{*}-\frac{i}{2} \xi_{\mu} \dot{\xi}^{\mu}-\frac{i}{2} \xi_{*} \dot{\xi}_{*}, \\
L_{B C L}=\frac{1}{2 e}\left(\dot{x}_{\mu}+i m^{-1} \dot{\xi}_{*} \xi_{\mu}\right)^{2}-\frac{e}{2} m^{2}-\frac{i}{2} \xi_{\mu} \dot{\xi}^{\mu}+\frac{i}{2} \xi_{*} \dot{\xi}_{*},
\end{gathered}
$$

where $x_{\mu}$ are even coordinates of the particle of mass $m, \xi_{\mu}$ and $\xi_{*}$ are Grassmann (odd) spin variables, $e$ and $\lambda$ are even and odd Lagrange multipliers, respectively, and we use the metric $\eta_{\mu \nu}=\operatorname{diag}(-,+, \ldots,+)$. Under space inversion the variables $e, \lambda$ and $\xi_{*}$ are transformed as a scalar, $P: e \rightarrow e$, and as pseudoscalars, $P: \xi_{*} \rightarrow-\xi_{*}, P: \lambda \rightarrow-\lambda$, whereas $x_{\mu}$ and $\xi_{\mu}$ are treated as vector and pseudovector quantities. In particular cases of $2+1$ and $3+1$ dimensions this correspondingly means that $P: x_{\mu} \rightarrow\left(x_{0},-x_{1}, x_{2}\right), P: \xi_{\mu} \rightarrow-\left(\xi_{0},-\xi_{1}, \xi_{2}\right)$ and $P: x_{\mu} \rightarrow\left(x_{0},-x_{1},-x_{2},-x_{3}\right), P: \xi_{\mu} \rightarrow-\left(\xi_{0},-\xi_{1},-\xi_{2},-\xi_{3}\right)$.

These models are usually referred to as one and the same model. Indeed, the Lagrangians can be equivalently represented as

$$
\begin{gathered}
L_{B M}=L_{0}-i\left(e^{-1} \dot{x} \xi-m \xi_{*}\right) \lambda, \\
L_{B C L}=L_{0}-i\left(e^{-1} \dot{x} \xi-m \xi_{*}\right) m^{-1} \dot{\xi}_{*},
\end{gathered}
$$

where

$$
L_{0}=\frac{1}{2 e} \dot{x}^{2}-\frac{e}{2} m^{2}-\frac{i}{2} \xi_{\mu} \dot{\xi}^{\mu}-\frac{i}{2} \xi_{*} \dot{\xi}_{*}
$$

Therefore, from here one could conclude that after identification

$$
\lambda=m^{-1} \dot{\xi}_{*}
$$


we have equivalent models. However, we shall see that this is not so: the non-equivalence of the models reveals itself in different and specific ways at the classical and quantum levels. Classically this becomes clear if we note that the BCL Lagrangian (2.2) unlike the Lagrangian of BM model (2.1) is quasi-invariant with respect to the transformations

$$
\xi_{*} \rightarrow \xi_{*}+\rho \Rightarrow L_{B C L} \rightarrow L_{B C L}+\frac{d}{d \tau}\left(\frac{i}{2} \rho \xi_{*}\right),
$$

where $\rho$ is an arbitrary odd real constant parameter. BCL Lagrangian, unlike that of BM model, is also quasi-invariant under the global transformations

$$
\delta x_{\mu}=-i m^{-1} \xi_{*} \epsilon_{\mu}, \quad \delta \xi_{\mu}=\epsilon_{\mu}, \quad \delta \xi_{*}=0
$$

with odd real constant vector $\epsilon_{\mu}$. The first Noether's theorem [13] gives us the associated scalar and vector integrals of motion being the generators of the transformations (2.7) and (2.8),

$$
\theta_{0}=\frac{1}{2} \xi_{*}+i \pi_{*}, \quad \Sigma_{\mu}=m \xi_{\mu}+\xi_{*} p_{\mu}
$$

where $\pi_{*}=\partial L_{B C L} / \partial \dot{\xi}_{*}$ and $p_{\mu}=\partial L_{B C L} / \partial \dot{x}^{\mu}$. The commutator of two supertranslations specified by the parameters $\rho_{1}, \epsilon_{1}^{\mu}$ and $\rho_{2}, \epsilon_{2}^{\mu}$ gives

$$
\delta_{1,2} x^{\mu}=i m^{-1}\left(\epsilon_{1}^{\mu} \rho_{2}-\epsilon_{2}^{\mu} \rho_{1}\right), \quad \delta_{1,2} \xi_{\mu}=\delta_{1,2} \xi_{*}=0,
$$

where $\delta_{1,2}=\delta_{2} \delta_{1}-\delta_{1} \delta_{2}$. Therefore, the commutator of supertransformations (2.7), (2.8) produces the space-time translation. This could be considered as an indication on some global space-time supersymmetry in BCL model. We shall discuss this point in detail in what follows.

\subsection{Relationship of the models: Lagrangian formalism}

Let us investigate the difference between BM and BCL systems in more detail. First, we shall show that classically BCL model has effectively one more odd dynamical variable in comparison with BM model, and this additional variable is a constant of motion generating the transformations (2.7). As a result, quantum theory of both models will not be equivalent in the case of even dimension of space-time, whereas in odd space-time dimension it will be the same. In spite of the quantum equivalence in latter case, we shall observe that BM model cannot reproduce classically some quantum symmetries being reproducible in BCL model.

Lagrange equations of motion of BCL model are

$$
\begin{gathered}
\dot{p}_{\mu}=0, \quad p^{2}+m^{2}=0, \\
\dot{\xi}_{\mu}+m^{-1} \dot{\xi}_{*} p_{\mu}=0, \\
\frac{d}{d \tau}\left(\xi_{*}-m^{-1} p \xi\right)=0
\end{gathered}
$$

where

$$
p_{\mu}=e^{-1}\left(\dot{x}_{\mu}+i m^{-1} \dot{\xi}_{*} \xi_{\mu}\right) .
$$

Equations of motion for BM model have the same form (2.11) but with $p_{\mu}$ given by

$$
p_{\mu}=e^{-1}\left(\dot{x}_{\mu}+i \lambda \xi_{\mu}\right) \text {, }
$$


whereas instead of Eqs. (2.12), (2.13) we have

$$
\begin{gathered}
\dot{\xi}_{\mu}+\lambda e^{-1} \dot{x}_{\mu}=0, \\
\dot{\xi}_{*}-m \lambda=0,
\end{gathered}
$$

and variation in $\lambda$ gives Lagrange constraint

$$
\xi_{*}-m^{-1} p \xi=0 .
$$

Solving Eq. (2.17) with respect to odd Lagrange multiplier, we arrive exactly at the relation (2.6) and conclude that energy-momentum vectors given by Eqs. (2.14) and (2.15) are identical, and as a consequence, Eqs. (2.12) are identical to Eqs. (2.16), whereas Eq. (2.13) is a derivative of Eq. (2.18). This means that for BM model we have the relation

$$
p \xi-m \xi_{*}=0
$$

whereas for BCL model,

$$
p \xi-m\left(\xi_{*}+\rho\right)=0,
$$

where $\rho$ is an arbitrary real odd constant in correspondence with symmetry (2.7) observed above.

Formally the classical non-equivalence happens here since for excluding Lagrange multiplier $\lambda$ from BM model we have to use the equation of motion for $\xi_{*}$ but not the equation for $\lambda$ itself (see the discussion of this point in Refs. [9, 3]).

\subsection{Hamiltonian formalism}

Let us consider the Hamiltonian description of the systems. The BM model is described by the canonical even variables $x_{\mu}, p^{\mu},\left\{x_{\mu}, p^{\nu}\right\}=\eta_{\mu}^{\nu}$, by $e, P_{e},\left\{e, P_{e}\right\}=1$, and by odd canonical variables $\xi_{\mu}$ and $\pi_{\mu},\left\{\xi_{\mu}, \pi_{\nu}\right\}=-\eta_{\mu \nu}, \xi_{*}$ and $\pi_{*},\left\{\xi_{*}, \pi_{*}\right\}=-1, \lambda$ and $\Pi_{\lambda},\left\{\lambda, \Pi_{\lambda}\right\}=-1$. First-class primary constraints are

$$
P_{e} \approx 0, \quad \Pi_{\lambda} \approx 0
$$

and second-class primary constraints are

$$
\begin{aligned}
& \pi_{*}-\frac{i}{2} \xi_{*} \approx 0, \\
& \pi_{\mu}-\frac{i}{2} \xi_{\mu} \approx 0
\end{aligned}
$$

Taking into account second-class constraints removes odd canonical momenta $\pi_{\mu}, \pi_{*}$, and gives the Dirac brackets

$$
\begin{gathered}
\left\{\xi_{\mu}, \xi_{\nu}\right\}=i \eta_{\mu \nu}, \\
\left\{\xi_{*}, \xi_{*}\right\}=i .
\end{gathered}
$$

Stability algorithm for the constraints (2.21) generates secondary constraints

$$
\phi=\frac{1}{2}\left(p^{2}+m^{2}\right) \approx 0, \quad \chi=p \xi-m \xi_{*} \approx 0 .
$$


The set of constraints (2.21), (2.26) is the set of first class constraints with constraints (2.26) forming $s(1)$ superalgebra [14]: $\{\chi, \chi\}=2 i \phi,\{\chi, \phi\}=0$. The total Hamiltonian [3], 13] is

$$
H_{t o t}=e \phi-i \lambda \chi+w P_{e}+\omega \Pi_{\lambda},
$$

where $w=w(\tau)$ and $\omega=\omega(\tau)$ are real even and odd arbitrary functions, respectively. Equations of motion generated by Hamiltonian (2.27),

$$
\begin{gathered}
\dot{p}_{\mu}=0, \quad \dot{x}_{\mu}=e p_{\mu}-i \lambda \xi_{\mu}, \quad \dot{e}=w \\
\dot{\xi}_{*}=m \lambda, \quad \dot{\xi}_{\mu}=-\lambda p_{\mu}, \quad \dot{\lambda}=\omega
\end{gathered}
$$

are equivalent to Lagrange equations of motion.

In the case of BCL model the primary constraints are $P_{e} \approx 0$,

$$
\chi=p \xi-m\left(\frac{1}{2} \xi_{*}-i \pi_{*}\right) \approx 0,
$$

and those given by Eq. (2.23). Taking into account second class constraints (2.23) excludes $\pi_{\mu}$ and gives again Dirac brackets (2.24). The condition $\dot{P}_{e}=0$ generates the mass shell constraint as a secondary constraint, and taking into account corresponding brackets, we find that constraints $\phi$ and $\chi$ form the same $s(1)$ superalgebra as $\phi$ and $\chi$ do in BM model. The total Hamiltonian

$$
H_{t o t}=e \phi+w P_{e}-i \tilde{\omega} \chi
$$

containing an arbitrary odd function $\tilde{\omega}=\tilde{\omega}(\tau)$ generates the equations of motion equivalent to Lagrange equations of motion. Let us define the real odd variables

$$
\theta_{0}=\frac{1}{2} \xi_{*}+i \pi_{*}, \quad \theta_{1}=\frac{1}{2} \xi_{*}-i \pi_{*}
$$

equivalent to the initial set of scalar odd variables $\xi_{*}$ and $\pi_{*}$. They have nontrivial Poisson brackets

$$
\left\{\theta_{\alpha}, \theta_{\beta}\right\}=i \eta_{\alpha \beta},
$$

where $\alpha, \beta=0,1, \eta_{\alpha \beta}=\operatorname{diag}(-,+)$. Therefore, odd constraint has the form

$$
\chi=p \xi-m \theta_{1} \approx 0 .
$$

Taking into account the form of the corresponding brackets and constraints as well as total Hamiltonians, one concludes that the Lagrange multiplier $\lambda$ of BM model can be identified with arbitrary odd function $\tilde{\omega}$ from BCL model, whereas the odd scalar space-like variable $\theta_{1}$

of the BCL model corresponds to the scalar variable $\xi_{*}$ of the BM model. Then the difference of the BCL model from the BM model consists in the presence of the additional time-like odd variable $\theta_{0}$ being a constant of motion which generates the transformation (2.7).

\subsection{Integrals of motion}

In addition to $\theta_{0}$, other classical integrals of motion of BCL model are the energy-momentum vector $p_{\mu}$, the total angular momentum tensor

$$
J_{\mu \nu}=x_{\mu} p_{\nu}-x_{\nu} p_{\mu}+i \xi_{\mu} \xi_{\nu}
$$


and odd vector

$$
\Xi_{\mu}=m \xi_{\mu}+\theta_{1} p_{\mu}
$$

However, not all the components of $\Xi_{\mu}$ are independent since taking into account the mass shell constraint, we have $p^{\mu} \Xi_{\mu} \approx m \chi \approx 0$. Due to the constraint $\chi \approx 0$, we have also the integral of motion $i \theta_{1} p \xi$ which classically (but not quantum mechanically) is weakly equal to zero. In the case of BM model all the listed integrals except $\theta_{0}$ (with corresponding change of $\theta_{1}$ for $\left.\xi_{*}\right)$ are the same.

As we stressed above, the integral $\theta_{0}$ generates in BCL model the symmetry transformations (2.7). On the other hand, the vector integral $\Sigma_{\mu}$ being the generator of supersymmetry transformations (2.8), is presented as

$$
\Sigma_{\mu}=\Xi_{\mu}+\theta_{0} p_{\mu}
$$

We see that the generator $\Sigma_{\mu}$ is the linear combination of the integral $\Xi_{\mu}$ and of the composition of odd, $\theta_{0}$, and even, $p_{\mu}$, integrals. Therefore, the integral $\Sigma_{\mu}$ plays no special independent role and the nature of the global supersymmetry (2.10) turns out to be trivial: it is encoded in the relation $\left\{\Sigma_{\mu}, \theta_{0}\right\}=-i p_{\mu}$ being a consequence of Eq. (2.36). Here we note that symmetry (2.8) presents also in BM model under taking into account the equation of motion (2.17). Its generator is the odd vector integral $\Xi_{\mu}=m \xi_{\mu}+\xi_{*} p_{\mu}$. Thus, we arrive once more at the conclusion that the essential difference of pseudoclassical BM model from the BCL model is due to the absence in it of the odd integral $\theta_{0}$.

To conclude the discussion of classical theory of BM and BCL models, we recall that according to the general theory of constrained dynamical systems [3, 13] any phase space function $A$ such that $\frac{d}{d \tau} A=0$ is the integral of motion, where $\tau$ is an evolution parameter. Any integral of motion can be considered as a generator of corresponding symmetry transformation. Observables are those phase space functions satisfying the relations $\left\{A, \phi_{a}\right\} \approx 0$, where $\phi_{a}$ is the set of constraints. In our case the Hamiltonians are linear combinations of constraints and, therefore, the observables are simultaneously integrals of motion.

\section{Quantization}

\subsection{Quantum equivalence of $3 D \mathrm{BM}$ and BCL models}

Let us consider the quantization of both models, and we start from the BM model. First we note that the pair of conjugate odd variables $\lambda$ and $\Pi_{\lambda}$ can be completely removed from the theory by introducing the gauge condition $\lambda-\lambda_{0} \approx 0$ to the constraint $\Pi_{\lambda} \approx 0$, where $\lambda_{0}$ is some odd constant. Then, constructing quantum analogs of odd variables $\xi_{\mu}$ and $\xi_{*}$ we should consider separately the cases of odd and even dimensions of space-time. We discuss here the most interesting case of 3-dimensional space-time. Other odd-dimensional cases can be considered in analogous way, whereas the case of even space-time dimension will be analyzed in Appendix.

The quantization of odd variables should give us irreducible representation of the Clifford algebra with 4 generators,

$$
\left[\widehat{\xi}_{\mu}, \widehat{\xi}_{\nu}\right]_{+}=-\eta_{\mu \nu}, \quad\left[\widehat{\xi}_{*}, \widehat{\xi}_{*}\right]_{+}=-1, \quad\left[\widehat{\xi}_{\mu}, \widehat{\xi}_{*}\right]_{+}=0
$$


which is 4-dimensional (in general case of $d=2 n-1$, the dimensionality of the corresponding Clifford algebra with $2 n$ generators is $2^{n}$ ). It is convenient to realize $\widehat{\xi}_{\mu}$ and $\widehat{\xi}_{*}$ as

$$
\widehat{\xi}_{\mu}=\frac{1}{\sqrt{2}} \gamma_{\mu} \otimes \sigma_{1}, \quad \widehat{\xi}_{*}=\frac{i}{\sqrt{2}} 1 \otimes \sigma_{2}
$$

where $(2+1)$-dimensional matrices $\gamma^{\mu}, \gamma^{0}=\sigma_{3}, \gamma^{j}=i \sigma_{j}, j=1,2$, satisfy the relation $\gamma^{\mu} \gamma^{\nu}=-\eta^{\mu \nu}+i \epsilon^{\mu \nu \lambda} \gamma_{\lambda}$ with totally antisymmetric tensor $\epsilon^{\mu \nu \lambda}$ normalized as $\epsilon^{012}=1$. The quantum analog of the odd constraint function is $\frac{1}{\sqrt{2}} 1 \otimes \sigma_{1} \cdot\left(p \gamma \otimes 1+m \cdot 1 \otimes \sigma_{3}\right)$, and therefore, the quantum constraint equation $\widehat{\chi} \Psi=0$ is equivalent to the pair of Dirac equations,

$$
\left(p \gamma \otimes 1+m \cdot 1 \otimes \sigma_{3}\right) \Psi=0,
$$

where we suppose that $\Psi$ is a doublet of spinor fields, presented in transposed form as $\Psi=\left(\psi_{u}, \psi_{d}\right), 1 \otimes \sigma_{3} \cdot \Psi=\left(\psi_{u},-\psi_{d}\right)$. Here and in what follows we denote the operators $p_{\mu}$ and $x_{\mu}$ in the same way as their classical counterparts. Eq. (3.2) generates the KleinGordon equation being the quantum analog of the constraint $\phi \approx 0$, and we conclude that the quantization of BM model in odd dimensional space-time gives the $P, T$-invariant system of two fermion fields.

Let us consider the quantum theory corresponding to the BCL model in odd-dimensional space-time. In this model we have the odd variable $\theta_{0}$ in addition to the variables $\xi_{\mu}$ and $\xi_{*}$ from BM model. The latter, as it was noted, should be identified with $\theta_{1}$. The quantum analog of additional odd variable can be realized as

$$
\widehat{\theta}_{0}=\frac{1}{\sqrt{2}} 1 \otimes \sigma_{3}
$$

whereas all other variables can be realized exactly as in BM model. Therefore, in $2+1$ dimensions the quantum analog of the odd constraint gives here, again, the $P, T$-invariant pair of Dirac equations. Since in BCL model we have the Clifford algebra with odd number of generators, in $2+1$ dimensions we have a relation being specific to its irreducible representations:

$$
2^{5 / 2} i \widehat{\theta}_{0} \widehat{\theta}_{1} \widehat{\xi}^{0} \widehat{\xi}^{1} \widehat{\xi}^{2}=1 .
$$

Therefore, using it, at the quantum mechanical level we can 'restore' the operator $\widehat{\theta}_{0}$ being absent from the BM model:

$$
\widehat{\theta}_{0}=2^{3 / 2} i \widehat{\xi}_{*} \widehat{\xi}^{0} \widehat{\xi}^{1} \widehat{\xi}^{2}
$$

Because of this, quantum mechanically BM and BCL models are equivalent in $(2+1)$ dimensional space-time as well as in any other odd space-time dimension. But since there is no classical analog of relation (3.4), classically these models cannot be considered as equivalent.

We conclude that BM and BCL models in $2+1$ dimensions describe the same quantum $P, T$-invariant system of two fermion fields.

\subsection{Scalar product}

Under quantization the scalar product should be introduced in such a way that all the quantum counterparts of classical real observables would be self-conjugate operators. In the 
case of BM model the choice $\left\langle\Psi_{1} \mid \Psi_{2}\right\rangle=\bar{\Psi}_{1} \Psi_{2}$ with $\bar{\Psi}=\Psi^{\dagger} \Delta, \Delta=\sqrt{2} \widehat{\xi}^{0}$, guarantees that the quantum operators corresponding to the observables $J_{\mu \nu}$ and $\Xi_{\mu}$ will be self-conjugate: $\left\langle\Psi_{1} \mid \widehat{O} \Psi_{2}\right\rangle^{*}=\left\langle\Psi_{2} \mid \widehat{O}^{\star} \Psi_{1}\right\rangle=\left\langle\Psi_{2} \mid \widehat{O} \Psi_{1}\right\rangle, \widehat{O}=\widehat{J}_{\mu \nu}, \widehat{\Xi}_{\mu}$. We find also that with such a choice the averaged odd quantum constraint gives the standard form for the field Lagrangian of the $3 D$ $P, T$-invariant fermion system:

$$
\langle\Psi \mid \widehat{\chi} \Psi\rangle=\Psi^{\dagger} \gamma^{0}\left(p \gamma \otimes 1+m 1 \otimes \sigma_{3}\right) \Psi=\mathcal{L} .
$$

Note that the operator $i \widehat{\theta}_{0}$ constructed according to the relation (3.5) is self-conjugate here but having no classical analog.

In the case of BCL model we find that the same indefinite scalar product with $\Delta=\sqrt{2} \widehat{\xi}^{0}$, guarantees that operators $\widehat{J}_{\mu \nu}$ and $\widehat{\Xi}_{\mu}$ are self-conjugate and that it produces the same field Lagrangian under averaging the quantum constraint $\widehat{\chi}$. But in this case we get that $\widehat{\theta}_{0}$, being observable, is an anti-self-conjugate operator. In the BCL model we cannot introduce the scalar product in such a way that all the quantum analogs of classical real observable quantities would be self-conjugate operators, and in this sense the model reveals some sort of quantum anomaly.

\section{CPV model}

\subsection{Classical theory}

The third pseudoclassical model to be considered here is the $3 D \mathrm{CPV}$ model [11] given by the Lagrangian

$$
L_{C P V}=\frac{1}{2 e}\left(\dot{x}_{\mu}+i v \epsilon_{\mu \nu \lambda} \xi^{\nu} \xi^{\lambda}\right)^{2}-\frac{e}{2} m^{2}-i m v \theta_{1} \theta_{2}-\frac{i}{2} \xi_{\mu} \dot{\xi}^{\mu}+\frac{i}{2} \theta_{a} \dot{\theta}_{a}
$$

with $v$ being even Lagrange multiplier instead of odd multiplier $\lambda$ taking place in BM model, and $\theta_{a}, a=1,2$, being the pair of odd Lorentz scalars. We assume that under $P$-inversions here, unlike the BM and BCL models, $\xi_{\mu}$ is transformed as vector $x_{\mu}, \theta_{1}$ is a scalar variable, whereas $\theta_{2}$ and $v$ are pseudoscalar quantities [6]. Due to the presence of even Lagrange multiplier instead of odd one the correspondence between CPV and BM (as well as BCL) models has a formal character given by relations $\lambda \xi_{\mu} \leftrightarrow v \epsilon_{\mu \nu \lambda} \xi^{\nu} \xi^{\lambda}, \lambda \xi_{*} \leftrightarrow v \theta_{1} \theta_{2}, \xi_{*} \dot{\xi}_{*} \leftrightarrow$ $-\theta_{a} \dot{\theta}_{a}$. The formal character of the relationship between models is clear also from the fact that CPV model, unlike BM and BCL models, has a global U(1) invariance,

$$
\theta^{ \pm} \rightarrow \theta^{\prime}=e^{ \pm i \omega} \theta^{ \pm}
$$

where $\theta^{ \pm}=\frac{1}{\sqrt{2}}\left(\theta_{1} \pm i \theta_{2}\right)$. As we shall see, this global invariance can be naturally localized in CPV model leading to the quantum $\mathrm{U}_{\sigma_{3}}(1)$ gauge symmetry [4, [6].

Dirac brackets for $\xi_{\mu}$ have the same form (2.24) as in BM and BCL models, whereas for $\theta_{a}$ we have

$$
\left\{\theta_{a}, \theta_{b}\right\}=-i \delta_{a b}
$$

The primary Hamiltonian constraints are $P_{e} \approx 0, P_{v} \approx 0$ with $P_{v}$ being canonical momentum for $v,\left\{v, P_{v}\right\}=1$. As a secondary constraint we get the mass shell constraint $\phi=\frac{1}{2}\left(p^{2}+\right.$ 
$\left.m^{2}\right) \approx 0$, and instead of odd constraint $\chi$ from BM and BCL models, we have here nonlinear in Grassmann variables even constraint

$$
\varrho=i \epsilon_{\mu \nu \lambda} p^{\mu} \xi^{\nu} \xi^{\lambda}-2 m i \theta_{1} \theta_{2} \approx 0
$$

The Hamiltonian is

$$
H=e \phi+v \varrho+u_{1} P_{e}+u_{2} P_{v},
$$

where $u_{1,2}=u_{1,2}(\tau)$ are arbitrary even functions. Due to nonlinearity of constraint (4.4) in Grassmann variables, it admits no gauge conditions. The general class of the systems with such peculiar constraints was investigated in Ref. [12].

\subsection{Integrals of motion}

The vectors $p_{\mu}$ and $J_{\mu}=-\frac{1}{2} \epsilon_{\mu \nu \lambda} J^{\nu \lambda}$, the latter having the same form as in two other models, are the integrals of motion. In addition, we have the following nilpotent integrals of motion: $\xi^{(0)}$ and

$$
i \theta_{1} \theta_{2}, \quad i \xi^{(1)} \xi^{(2)}, \quad i \xi^{(i)} \theta^{i}, \quad i \epsilon_{i j} \xi^{(i)} \theta^{j}
$$

where $\theta^{i}=\theta_{i}, i, j=1,2, \xi^{(\alpha)}=\xi^{\mu} e_{\mu}^{(\alpha)}, \alpha=0,1,2, \epsilon_{i j}=-\epsilon_{j i}, \epsilon_{12}=1$. Here having in mind the mass shell constraint, we introduced the complete oriented triad $e_{\mu}^{(\alpha)}=e_{\mu}^{(\alpha)}(p)$, $\alpha=0,1,2$,

$$
e_{\mu}^{(0)}=\frac{p_{\mu}}{\sqrt{-p^{2}}}, \quad e_{\mu}^{(\alpha)} \eta_{\alpha \beta} e_{\nu}^{(\beta)}=\eta_{\mu \nu}, \quad \epsilon_{\mu \nu \lambda} e^{(0) \mu} e^{(i) \nu} e^{(j) \lambda}=\epsilon^{0 i j} .
$$

Note that unlike $e_{\mu}^{(0)}$, the components $e_{\mu}^{(i)}$ are non-covariant objects and as a result, the same is true for the quantities $\xi^{(i)}$.

In the case of $2+1$ dimensions in BM and BCL models we have two independent components of odd vector integral (2.35) and one even nilpotent integral $i \xi^{(0)} \theta_{1} \approx 0$, which are supplemented by one odd scalar integral $\theta_{0}$ in BCL model. In contrast, the CPV model has only one odd scalar integral $\xi^{(0)}$ and the set of four even nilpotent integrals (4.5), two of which on mass shell are related by the nilpotent constraint (4.4): $i \xi^{(1)} \xi^{(2)}-i \theta_{1} \theta_{2} \approx 0$.

\subsection{Quantization}

The quantum counterparts of odd variables satisfying the anticommutation relations $\left[\widehat{\theta}_{a}, \widehat{\theta}_{b}\right]_{+}=\delta_{a b},\left[\widehat{\xi}_{\mu}, \widehat{\xi}_{\nu}\right]_{+}=-\eta_{\mu \nu}$ and $\left[\widehat{\xi}_{\mu}, \widehat{\theta}_{a}\right]_{+}=0$ can be realized as

$$
\widehat{\xi}^{\mu}=\frac{1}{\sqrt{2}} \gamma^{\mu} \otimes \sigma_{3}, \quad \widehat{\theta}_{a}=\frac{1}{\sqrt{2}} 1 \otimes \sigma_{a} .
$$

Nilpotent constraint (4.4) turns into the equation

$$
\left(p \gamma \otimes 1+m \cdot 1 \otimes \sigma_{3}\right) \Psi=0
$$

which itself produces the mass shell (Klein-Gordon) equation. The scalar product

$$
\left\langle\Psi_{1} \mid \Psi_{2}\right\rangle=\Psi_{1}^{\dagger} \gamma^{0} \otimes 1 \Psi_{2}
$$


gives the relation of the form (3.6), $\frac{1}{2}\langle\Psi \mid \widehat{\varrho} \Psi\rangle=\mathcal{L}$, and we conclude that quantum mechanically CPV and $3 D$ BM and BCL models are equivalent: all of them result in the same $3 D$ $P, T$-invariant fermion system. However it is necessary to note that unlike the BCL model, all the odd operators of CPV model, $\widehat{\xi}_{\mu}$ and $\widehat{\theta}_{a}$, are self-conjugate. This difference is coded in different nature of classical counterparts of operators $\widehat{\theta}_{a}$ and $\widehat{\theta}_{\alpha}$ : variables $\theta_{a}, a=1,2$, from CPV model are characterized by the definite metric $\delta_{a b}$ in the sense of brackets (4.3), whereas brackets (2.33) for variables $\theta_{\alpha}, \alpha=0,1$, from BCL model contain the indefinite metric $\eta_{\alpha \beta}$. As we shall see in Section 6, as a consequence of this formal difference in properties of odd operators, not all the classical analogs of corresponding quantum symmetry generators will be real quantities in 3D BCL and BM models. On the other hand, even character of classical counterpart (4.4) of quantum constraint (4.7) will be the obstacle for reproducing classical analog of a part of quantum symmetries in CPV model.

\section{Quantum symmetries}

Let us describe the global symmetries of the quantum $3 D P, T$-invariant fermion system given by Eq. (4.7), by the corresponding field Lagrangian $\mathcal{L}=\Psi^{\dagger} \gamma^{0} \otimes 1\left(p \gamma \otimes 1+m \cdot 1 \otimes \sigma_{3}\right) \Psi$ and associated Dirac scalar product (4.8). Eq. (4.7) is equivalent to the Klein-Gordon equation and to the equation $\Pi_{+} \Psi=0$ with the operator $\Pi_{+}=\frac{1}{2}\left(1+\gamma^{(0)} \otimes \sigma_{3}\right)$ being the projector operator, $\Pi_{+}^{2}=\Pi_{+}$. The complete set of observable operators is the set of operators being self-conjugate with respect to the scalar product (4.8) and commuting with Klein-Gordon operator $p^{2}+m^{2}$ and with the operator $\Pi_{+}$. This is the set of operators $p_{\mu}$, $\widehat{J}_{\mu}=-\epsilon_{\mu \nu \lambda} x^{\nu} p^{\lambda}-\frac{1}{2} \gamma_{\mu} \otimes 1$, and

$$
\widehat{S}=-\frac{1}{2} \gamma^{(0)} \otimes 1, \quad \widehat{N}=\frac{1}{2} 1 \otimes \sigma_{3}, \quad \widehat{T}^{i}=-\frac{1}{2} \gamma^{(i)} \otimes \sigma_{1}, \quad \widehat{K}^{i}=-\frac{1}{2} \gamma^{(i)} \otimes \sigma_{2} .
$$

Though operators $\widehat{T}^{i}$ and $\widehat{K}^{i}$ are related by unitary transformation, nevertheless the usage of both sets of operators will be necessary in what follows. Operators $\widehat{\mathcal{W}}^{\alpha}, \alpha=0,1$, 2, where $\widehat{\mathcal{W}}^{0}=\widehat{S}$, and $\widehat{\mathcal{W}}^{i}=\widehat{T}^{i}$, or $\widehat{\mathcal{W}}^{i}=\widehat{K}^{i}$, and operators $\widehat{\mathcal{N}}^{\alpha}$, where $\widehat{\mathcal{N}}^{0}=-\widehat{\mathcal{N}}$ and $\widehat{\mathcal{N}}^{1}=\widehat{T}^{1}$, $\widehat{\mathcal{N}}^{2}=\widehat{K}^{1}$, or $\widehat{\mathcal{N}}^{1}=\widehat{T}^{2}, \widehat{\mathcal{N}}^{2}=\widehat{K}^{2}$, form $\operatorname{su}(1,1)$ algebras,

$$
\left[\widehat{\mathcal{W}}_{\alpha}, \widehat{\mathcal{W}}_{\beta}\right]=-i \epsilon_{\alpha \beta \gamma} \widehat{\mathcal{W}}^{\gamma}, \quad\left[\widehat{\mathcal{N}}_{\alpha}, \widehat{\mathcal{N}}_{\beta}\right]=-i \epsilon_{\alpha \beta \gamma} \widehat{\mathcal{N}}^{\gamma}
$$

Simultaneously, they are generators of Clifford algebras $C l_{1,2}$,

$$
\left[\widehat{\mathcal{W}}_{\alpha}, \widehat{\mathcal{W}}_{\beta}\right]_{+}=-\frac{1}{2} \eta_{\alpha \beta}, \quad\left[\widehat{\mathcal{N}}_{\alpha}, \widehat{\mathcal{N}}_{\beta}\right]_{+}=-\frac{1}{2} \eta_{\alpha \beta}
$$

The projector operator $\Pi_{-}=1-\Pi_{+}$commutes with all the observable operators (5.1). As a result, one can construct operators $\widehat{\mathcal{R}}_{\alpha}=\Pi_{-} \widehat{\mathcal{W}}_{\alpha}$ and $\widehat{\mathcal{U}}_{\alpha}=\Pi_{-} \widehat{\mathcal{N}}_{\alpha}$ forming the $s u(1,1)$ algebras but giving the $s(1,2)$ superalgebras [14

$$
\begin{aligned}
{\left[\widehat{\mathcal{R}}_{\alpha}, \widehat{\mathcal{R}}_{\beta}\right]_{+} } & =-\frac{1}{2} \eta_{\alpha \beta} \Pi_{-}, \quad\left[\Pi_{-}, \widehat{\mathcal{R}}_{\alpha}\right]=0, \\
{\left[\widehat{\mathcal{U}}_{\alpha}, \widehat{\mathcal{U}}_{\beta}\right]_{+} } & =-\frac{1}{2} \eta_{\alpha \beta} \Pi_{-}, \quad\left[\Pi_{-}, \widehat{\mathcal{U}}_{\alpha}\right]=0
\end{aligned}
$$


instead of Clifford algebras $C l_{1,2}$. Operators $\widehat{\mathcal{R}}_{\alpha}$ were revealed in Ref. [6] as integrals of motion of $P, T$-invariant planar fermion system:

$$
\begin{gathered}
\widehat{\mathcal{R}}^{0}=\frac{1}{2}(\widehat{S}+\widehat{N})=-\frac{1}{4}\left(\gamma^{(0)} \otimes 1-1 \otimes \sigma_{3}\right), \\
\widehat{\mathcal{R}}^{1}=\frac{1}{2}\left(K^{(1)}-T^{(2)}\right)=\frac{1}{4}\left(\gamma^{(2)} \otimes \sigma_{1}-\gamma^{(1)} \otimes \sigma_{2}\right), \\
\widehat{\mathcal{R}}^{2}=\frac{1}{2}\left(K^{(2)}+T^{(1)}\right)=-\frac{1}{4}\left(\gamma^{(1)} \otimes \sigma_{1}+\gamma^{(2)} \otimes \sigma_{2}\right) .
\end{gathered}
$$

Operator $\Pi_{-}$turns into unity on the physical subspace (4.7). As a result, operators $\widehat{\mathcal{R}}_{\alpha}$ and $\widehat{\mathcal{U}}_{\alpha}$, like $\widehat{\mathcal{W}}_{\alpha}$ and $\widehat{\mathcal{N}}_{\alpha}$, generate spin-1/2 representation of $\mathrm{SU}(1,1)$.

One could conclude that the described symmetries (generated by the corresponding observable operators) are trivial. Indeed, $\widehat{S}$ is the spin operator related to $\widehat{N}$ via the basic equation (4.7) and $\widehat{T}^{i}, \widehat{K}^{i}$ simply mix 'up' ( $\left.\operatorname{spin} s=-1 / 2\right)$ and 'down' ( $\operatorname{spin} s=+1 / 2$ ) states. However, the non-triviality of these symmetries consists in the fact that the operators $\widehat{T}^{i}$ and $\widehat{K}^{i}$ are not Lorentz scalars. Their covariant counterparts can be presented, e.g., in the vector form

$$
\tilde{\mathcal{T}}_{\mu}=\epsilon_{\mu \nu \lambda} p^{\nu} \gamma^{\lambda} \otimes \sigma_{1}=\frac{\sqrt{2}}{m} \epsilon_{\mu \nu \lambda} p^{\nu} \widehat{\Xi}^{\lambda}, \quad \tilde{\mathcal{K}}_{\mu}=\epsilon_{\mu \nu \lambda} p^{\nu} \gamma^{\lambda} \otimes \sigma_{2}=2 i \tilde{\mathcal{T}}_{\mu} \widehat{N}
$$

$p_{\mu} \tilde{\mathcal{T}}^{\mu}=p_{\mu} \tilde{\mathcal{K}}^{\mu}=0$. These (related by $\widehat{N}$ ) covariant operators act non-trivially in the fermion spaces specified by the corresponding (omitted) spinor indices of $\psi_{u}$ and $\psi_{d}$ and in 'isotopic' space described by $u, d$ subindices, and being linear in $p_{\mu}$, act also on the space-time argument of the state $\Psi(x)$. Their (anti)commutators,

$$
\left[\tilde{\mathcal{T}}_{\mu}, \tilde{\mathcal{T}}_{\nu}\right]=2\left(p^{2} \eta_{\mu \nu}-p_{\mu} p_{\nu}\right), \quad\left[\tilde{\mathcal{T}}_{\mu}, \tilde{\mathcal{T}}_{\nu}\right]_{+}=4 i \epsilon_{\mu \nu \lambda} p^{\lambda}(p \widehat{J})
$$

are nonlinear in Poincaré generators.

Actually, as was noted in Refs. [5, 6], the $P, T$-invariant fermion system can be considered as the system realizing irreducible representation of the nonstandard super-extension of the $3 D$ Poincaré group characterized by zero superspin. Let us describe such super-extension associated to the $P, T$-invariant planar fermion system. Here we have two related possibilities in correspondence with non-covariant (super)algebraic relations described above. First, the generators of $3 D$ Poincaré group, $p_{\mu}$ and $\widehat{J}_{\mu}$, can be supplemented by the generators $\tilde{\mathcal{W}}_{\mu}=$ $e_{\mu}^{(\alpha)}(p) \widehat{W}_{\alpha}$ (or by the generators $\tilde{\mathcal{N}}_{\mu}=e_{\mu}^{(\alpha)}(p) \widehat{N}_{\alpha}$ ). Then in addition to the $3 D$ Poincaré algebra, $\left[p_{\mu}, p_{\nu}\right]=0,\left[\widehat{J}_{\mu}, \widehat{J}_{\nu}\right]=-i \epsilon_{\mu \nu \lambda} \widehat{J}^{\lambda},\left[\widehat{J}_{\mu}, p_{\nu}\right]=-i \epsilon_{\mu \nu \lambda} p^{\lambda}$, we have the (anti)commutation relations

$$
\begin{gathered}
{\left[\tilde{\mathcal{W}}_{\mu}, \tilde{\mathcal{W}}_{\nu}\right]=-i \epsilon_{\mu \nu \lambda} \tilde{\mathcal{W}}^{\lambda}, \quad\left[\tilde{\mathcal{W}}_{\mu}, \tilde{\mathcal{W}}_{\nu}\right]_{+}=-\frac{1}{2} \eta_{\mu \nu}} \\
{\left[\widehat{J}_{\mu}, \tilde{\mathcal{W}}_{\nu}\right]=-i \epsilon_{\mu \nu \lambda} \tilde{\mathcal{W}}^{\lambda}, \quad\left[p_{\mu}, \tilde{\mathcal{W}}_{\nu}\right]=0,}
\end{gathered}
$$

This gives us the super-extension of the Poincaré algebra characterized by the Casimir operators $p^{2}$ and $\mathcal{S}=e_{\mu}^{(0)} \mathcal{J}^{\mu}, \mathcal{J}_{\mu}=\widehat{J}_{\mu}-\tilde{\mathcal{W}}_{\mu},\left[\mathcal{J}_{\mu}, \mathcal{J}_{\nu}\right]=-i \epsilon_{\mu \nu \lambda} \mathcal{J}^{\lambda}$. The operator $\mathcal{S}$ has a sense of superspin. Under taking into account the concrete realization of $\tilde{\mathcal{W}}_{\mu}$, we find that it identically turns into zero. 
Another possibility to construct the super-extension of the Poincare algebra consists in supplementing the set of Poincaré generators by the covariant set of generators $\Pi_{-}$and $\tilde{\mathcal{R}}_{\mu}$ (or by $\tilde{\mathcal{U}}_{\mu}$ instead of $\tilde{\mathcal{R}}_{\mu}$ ), satisfying the superalgebraic relations

$$
\begin{gathered}
{\left[\tilde{\mathcal{R}}_{\mu}, \tilde{\mathcal{R}}_{\nu}\right]=-i \epsilon_{\mu \nu \lambda} \tilde{\mathcal{R}}^{\lambda}, \quad\left[\tilde{\mathcal{R}}_{\mu}, \tilde{\mathcal{R}}_{\nu}\right]_{+}=-\frac{1}{2} \Pi_{-} \eta_{\mu \nu}, \quad\left[\tilde{\mathcal{R}}_{\mu}, \Pi_{-}\right]=0,} \\
{\left[\widehat{J}_{\mu}, \tilde{\mathcal{R}}_{\nu}\right]=-i \epsilon_{\mu \nu \lambda} \tilde{\mathcal{R}}^{\lambda}, \quad\left[p_{\mu}, \tilde{\mathcal{R}}_{\nu}\right]=\left[\Pi_{-}, p_{\mu}\right]=\left[\Pi_{-}, \widehat{J}_{\mu}\right]=0 .}
\end{gathered}
$$

Here $\tilde{\mathcal{R}}_{\mu}=e_{\mu}^{(\alpha)}(p) \widehat{R}_{\alpha}$ and $\Pi_{-}=\frac{1}{2}+2 \widehat{S} \widehat{N}$. In this case the superspin Casimir operator is given by $\mathcal{S}=e_{\mu}^{(0)} \mathcal{J}^{\mu}$, with $\mathcal{J}_{\mu}=\widehat{J}_{\mu}-\tilde{\mathcal{R}}_{\mu}$. The superspin takes here zero value on the physical subspace specified by Eq. (4.7). This second form of super-extension of the Poincaré algebra was discussed in Refs. [5, 6] as a hidden supersymmetry of the $P, T$-invariant planar fermion system.

Having in mind that in $2+1$ dimensions the transformations of space inversion are realized as [6] $P: \Psi(x) \rightarrow \Psi^{\prime}\left(x^{\prime}\right)=\eta U_{P} \Psi(x), U_{P}=\gamma^{1} \otimes \sigma_{1}, x_{\mu}^{\prime}=\left(x_{0},-x_{1}, x_{2}\right),|\eta|=1$, we find that the operator $\widehat{N}$ is parity-odd, $\left[\widehat{N}, U_{P}\right]_{+}=0$, and, being a Lorentz scalar, has a sense of changing parity operator. The given quantum $P$-transformations correspond to the classical transformations of space inversion accepted in BM, BCL and CPV models.

\section{Classical symmetries and anomalies}

Let us find the classical even and odd integrals of motion corresponding to quantum observables. For BCL model the correspondence is the following:

$$
\begin{gathered}
\widehat{S} \rightarrow S_{e}=-\frac{i}{2} \epsilon_{i j} \xi^{(i)} \xi^{(j)}, \quad S_{o}=-\sqrt{2} \theta_{0} \theta_{1} \xi^{(0)}, \\
\widehat{N} \rightarrow N_{e}=-\frac{i}{3} \theta_{1} \epsilon_{\mu \nu \lambda} \xi^{\mu} \xi^{\nu} \xi^{\lambda}, \quad N_{o}=\frac{1}{\sqrt{2}} \theta_{0}, \\
\widehat{T}^{i} \rightarrow T_{e}^{i}=-2 i \theta_{0} \theta_{1} \xi^{(0)} \epsilon^{i j} \xi^{(j)}, \quad T_{o}^{i}=-\frac{1}{\sqrt{2}} \xi^{(i)}, \\
\widehat{K}^{i} \rightarrow K_{e}^{i}=i \theta_{0} \xi^{(i)}, \quad K_{o}^{i}=-\sqrt{2} \theta_{1} \xi^{(0)} \epsilon^{i j} \xi^{(j)} .
\end{gathered}
$$

One can check that $S_{e}$ and $K_{e}^{i}$ reproduce $s u(1,1)$ algebra with respect to classical brackets. The classical integrals $N_{o}$ and $T_{o}^{i}$ reproduce classically the analog of $C l_{1,2}$ algebra. So, classically, unlike the quantum case, $s u(1,1)$ and $C l_{1,2}$ in BCL model are generated by different sets of integrals of motion. On the other hand, though $S_{e}$ and $T_{e}^{i}$ commute with respect to the brackets in appropriate way, the quantities $T_{e}^{i}, i=1,2$, satisfy the relation $\left\{T_{e}^{1}, T_{e}^{2}\right\}=0$, and therefore, this set does not reproduce classically $s u(1,1)$. Note also that $S_{o}, N_{e}, T_{e}^{i}$ and $K_{o}^{i}$ are pure imaginary classical quantities. Thus, we can reproduce here classically $s u(1,1)$ and $C l_{1,2}$, but, nevertheless, we cannot reproduce all the (anti)commutation relations of the corresponding quantum integrals. The asymmetry between classical analogs of operators $\widehat{T}^{i}$ and $\widehat{K}^{i}$ has a simple reason: though the basic equation (4.7) is 'symmetric' in indices $a=1,2$ of $\sigma$-matrices, the concrete realization of odd operators (3.1) in the model destroys this symmetry. 
Let us multiply classical generators of $s u(1,1)$ and $C l_{1,2}$ by $C=\frac{1}{2}+\xi^{(0)} \theta_{1}$, which commutes with all the classical integrals of motion listed above, i.e. is a central element weakly equal to $\frac{1}{2}$. As a result we get classically $s(1,2)$ superalgebra instead of Clifford algebra $C l_{1,2}$, but destroy $s u(1,1)$ bracket relations of even generators since $C$ being the classical analog of the operator $\Pi_{-}$does not reproduce its defining property $\Pi_{-}^{2}=\Pi_{-}$. Therefore, though in BCL model we can reproduce classically $s(1,2)$ superalgebra, its generators, unlike the quantum generators of $s(1,2)$ superalgebra, are not related at all to $s u(1,1)$ generators.

As we remember, the BM model is different from the BCL model because of absence of classical odd integral $\theta_{0}$. Since the sets of even, $S_{e}, K_{e}^{i}$, and odd, $N_{o}, T_{o}^{i}$, integrals of motion of BCL model contain $\theta_{0}$, classically either $s u(1,1)$ or $C l_{1,2}$ cannot be reproduced in BM model. So, in BM model even a part of quantum symmetries cannot be reproduced classically.

Let us turn to the CPV model. Here formally the direct classical analogs of all the listed above quantum observables can be constructed:

$$
\begin{gathered}
\widehat{S} \rightarrow S_{e}=-\frac{i}{2} \epsilon_{i j} \xi^{(i)} \xi^{(j)}, \quad S_{o}=i \sqrt{2} \theta_{1} \theta_{2} \xi^{(0)}, \\
\widehat{N} \rightarrow N_{e}=-i \theta_{1} \theta_{2}, \quad N_{o}=-\frac{i}{3 \sqrt{2}} \epsilon_{\mu \nu \lambda} \xi^{\mu} \xi^{\nu} \xi^{\lambda}, \\
\widehat{T}^{i} \rightarrow T_{e}^{i}=i \theta_{2} \xi^{(i)}, \quad T_{o}^{i}=-i \sqrt{2} \theta_{1} \xi^{(0)} \epsilon^{i j} \xi^{(j)}, \\
\widehat{K}^{i} \rightarrow K_{e}^{i}=-i \theta_{1} \xi^{(i)}, \quad K_{o}^{i}=-i \sqrt{2} \theta_{2} \xi^{(0)} \epsilon^{i j} \xi^{(j)}
\end{gathered}
$$

All these quantities are real, but not all of them are classical integrals of motion: $T_{e}^{i}, T_{o}^{i}, K_{e}^{i}$ and $K_{o}^{i}$ are not conserved. Only their specific linear combinations, $K_{e}^{1}-T_{e}^{2}, K_{e}^{2}+T_{e}^{1}$, and $K_{o}^{1}-T_{o}^{2}, K_{o}^{2}+T_{o}^{1}$, are integrals of motion. One can check that even linear combinations $\frac{1}{2}\left(K_{e}^{1}-T_{e}^{2}\right), \frac{1}{2}\left(K_{e}^{2}+T_{e}^{1}\right)$, together with even linear combination $\frac{1}{2}\left(S_{e}+N_{e}\right)$, being classical analogs of quantum linear combinations (5.2), reproduce classically $s u(1,1)$. On the other hand, the odd linear combinations $\frac{1}{2}\left(K_{o}^{1}-T_{o}^{2}\right)$ and $\frac{1}{2}\left(K_{o}^{2}+T_{o}^{1}\right)$, together with odd linear combination $\frac{1}{2}\left(S_{o}+N_{o}\right)$ reproduce classical analog of $s(1,2)$ superalgebra. Note that these classical generators of $s(1,2)$ are simply $s u(1,1)$ generators multiplied by the odd integral $-\sqrt{2} \xi^{(0)}$. At the quantum level the same operators correspond to even and odd abovementioned integrals of motion, and, as a consequence, operators $\widehat{\mathcal{R}}_{\alpha}$ satisfy simultaneously $s u(1,1)$ commutation and $s(1,2)$ anticommutation relations.

Therefore, we see that the CPV model reproduces classically $s u(1,1)$ and $s(1,2)$, but fails to reproduce $C l_{1,2}$. What is a reason of such difference between quantum and classical case in this model? In quantum case the observables $\widehat{T}^{i}$ and $\widehat{K}^{i}$ anticommute with the quantum operator corresponding to the classical constraint $\varrho$. Therefore, from this point of view, they form some sort of superalgebra with $\widehat{\varrho}$. But classically $\varrho$ is the even quantity. Due to this, the quantum anticommutation relations of $\widehat{T}^{i}$ and $\widehat{K}^{i}$ with $\widehat{\varrho}$ cannot be reproduced classically with respect to the bracket. This results in the fact that only part of quantum observables has classical analogs and, as a consequence, not all the quantum symmetry superalgebraic relations can be reproduced classically in CPV model. 


\section{$7 \quad \mathrm{U}_{\sigma_{3}}(1)$ gauge symmetry}

As we noted, the CPV model is invariant under global $U(1)$ transformations (4.2) generated by the integral $N_{\theta}=-i \theta_{1} \theta_{2}$. Gauging this symmetry, $\omega \rightarrow \omega\left(x_{\mu}(\tau)\right)$, we arrive at the Lagrangian [6]

$$
L_{C P V}^{g}=L_{C P V}+q N_{\theta}\left(\dot{x}_{\mu} \mathcal{A}^{\mu}+\frac{e}{2} i \xi_{\mu} \xi_{\nu} \mathcal{F}^{\mu \nu}\right)
$$

being invariant with respect to the local U(1) transformations:

$$
\theta^{ \pm} \rightarrow \theta^{ \pm \prime}=e^{ \pm i \omega(x)} \theta^{ \pm}, \quad \mathcal{A}_{\mu} \rightarrow \mathcal{A}_{\mu}^{\prime}=\mathcal{A}_{\mu}-q^{-1} \partial_{\mu} \omega(x)
$$

Here $\mathcal{A}_{\mu}=\mathcal{A}_{\mu}(x)$ is a $\mathrm{U}(1)$ gauge field, $q$ is a coupling constant and $\mathcal{F}_{\mu \nu}=\partial_{\mu} \mathcal{A}_{\nu}-\partial_{\nu} \mathcal{A}_{\mu}$. Lagrangian (7.1) leads to the modified (gauged) constraints

$$
\phi^{g}=\frac{1}{2}\left(\mathcal{P}^{2}+m^{2}-i q N_{\theta} \xi_{\mu} \xi_{\nu} \mathcal{F}^{\mu \nu}\right) \approx 0, \quad \varrho^{g}=i \epsilon_{\mu \nu \lambda} \mathcal{P}^{\mu} \xi^{\nu} \xi^{\lambda}-2 m N_{\theta} \approx 0,
$$

where $\mathcal{P}_{\mu}=p_{\mu}-q N_{\theta} \mathcal{A}_{\mu}$. These constraints form the same trivial algebra, $\left\{\phi^{g}, \varrho^{g}\right\}=0$, which takes place in a free case. The quantum counterparts of classical constraints (7.2) have the form

$$
\begin{gathered}
\widehat{\varrho}^{g} \Psi=0, \quad \widehat{\phi}^{g} \Psi=0, \\
\widehat{\varrho}^{g}=\widehat{\mathcal{P}} \gamma \otimes 1+m \cdot 1 \otimes \sigma_{3}, \quad 2 \widehat{\phi}^{g}=\widehat{\mathcal{P}}^{2}+m^{2}+\frac{1}{4} q \epsilon_{\mu \nu \lambda} \mathcal{F}^{\mu \nu} \gamma^{\lambda} \otimes 1,
\end{gathered}
$$

where $\widehat{\mathcal{P}}_{\mu}=p_{\mu}-\frac{1}{2} q \mathcal{A}_{\mu}(x) 1 \otimes \sigma_{3}$. The quantum constraints reproduce the trivial algebra, $\left[\widehat{\phi}^{g}, \widehat{\varrho}^{g}\right]=0$, and as in a free case, satisfy the relation $\widehat{\varrho}^{g 2}=-2 \widehat{\phi}^{g}+2 m\left(1 \otimes \sigma_{3}\right) \widehat{\varrho}^{g}$ which says that the gauged mass shell condition is a consequence of the gauged constraint $\widehat{\varrho}^{g}$. The explicit form of the quantum constraints (7.4) means that the $\mathrm{U}_{\sigma_{3}}(1)$ gauge interaction is the usual U(1) gauge interaction with the only difference that spin $s=-1 / 2$ and $s=+1 / 2$ states have the coupling constants of the opposite sign. This specific form of U(1) gauge interaction was used for modelling high-temperature superconductivity 4 . Thus, we conclude that the localization of the global $\mathrm{U}(1)$ symmetry of CPV model gives in a natural way the $\mathrm{U}_{\sigma_{3}}(1)$ gauge theory for $P, T$-invariant planar fermion system.

There is no natural analog of global $\mathrm{U}(1)$ symmetry (4.2) in BM and BCL models. To reproduce classically $\mathrm{U}_{\sigma_{3}}(1)$ gauge symmetry in these models, we construct direct classical analogs of quantum constraint operators (7.4). The necessary modification of odd constraint in BM model is

$$
\chi^{g}=p \xi+\frac{i}{2} q \epsilon_{\mu \nu \lambda} \mathcal{A}^{\mu} \xi^{\nu} \xi^{\lambda} \xi_{*}-m \xi_{*} \approx 0 .
$$

In BCL model the modified constraint has the same form with the change $\xi_{*}$ for $\theta_{1}$. The quantum analog of constraint (7.5) is exactly the first quantum equation from Eqs. (7.3) (multiplied by $\frac{1}{\sqrt{2}} 1 \otimes \sigma_{1}$ ). To find the modified even constraint, we calculate the bracket of odd constraint with itself, $\left\{\chi^{g}, \chi^{g}\right\}=2 i \tilde{\phi}$, and get

$$
2 \tilde{\phi}=p^{2}+\left(m-\frac{i}{2} q \epsilon_{\mu \nu \lambda} \mathcal{A}^{\mu} \xi^{\nu} \xi^{\lambda}\right)^{2}-\frac{1}{3} q \epsilon_{\mu \nu \lambda} \xi^{\mu} \xi^{\nu} \xi^{\lambda} \xi_{*} \partial_{\sigma} \mathcal{A}^{\sigma}-i q \epsilon_{\mu \nu \lambda}\left(p^{\mu} \mathcal{A}^{\nu}+\mathcal{A}^{\nu} p^{\mu}\right) \xi^{\lambda} \xi_{*} \approx 0
$$


Due to Jacobi identity we have $\left\{\chi^{g}, \tilde{\phi}\right\}=0$ and conclude that the modified constraints form the same $s(1)$ superalgebra as in a free case. Note that in BM and BCL models, unlike the CPV model, classical even constraint contains no term proportional to $\mathcal{F}_{\mu \nu}$. This term appears only quantum mechanically, under construction of the quantum analog of the constraint (7.6). Indeed, choosing the same ordering for quantum counterparts of the classical quantities presenting in Eq. (7.6), we get

$$
2 \widehat{\tilde{\phi}}=2 \widehat{\phi}^{g}-q \mathcal{A} \gamma \otimes \sigma_{3} \cdot \widehat{\varrho}^{g},
$$

where $\widehat{\phi}^{g}$ and $\widehat{\varrho}^{g}$ are given by Eq. (7.4). Let us stress that to get the correct form of the quantum constraint $\widehat{\tilde{\phi}}$, it is essential to take into account the term $-\frac{1}{4} q^{2}\left(\epsilon_{\mu \nu \lambda} \mathcal{A}^{\mu} \xi^{\nu} \xi^{\lambda}\right)^{2}$ which appears from the second term in Eq. (7.6) and classically is equal to zero. Moreover, for getting the appropriate quantum analog of even constraint, it is essential to preserve in (7.6) the term $\frac{1}{3} q \epsilon_{\mu \nu \lambda} \xi^{\mu} \xi^{\nu} \xi^{\lambda} \xi_{*} \partial_{\sigma} \mathcal{A}^{\sigma}$ in spite of the fact that classically it is proportional to $\chi^{g}$, and so, itself is weakly equal to zero.

From here we conclude that the quantization of even constraint gives the necessary gauged quantum mass shell condition, but the relationship of classical and quantum theories for the $\mathrm{U}_{\sigma_{3}}(1)$ gauged BM and BCL models is not direct and natural.

Concluding the discussion of classical $\mathrm{U}_{\sigma_{3}}(1)$ gauge symmetry, let us write explicitly the corresponding modified Lagrangians for BM and BCL models which can be obtained by inverse Legendre transformation proceeding from the classical constraints (7.5) and (7.6):

$$
\begin{gathered}
L_{B M}^{g}=\frac{1}{2 e}\left(\dot{x}_{\mu}-i \lambda \xi_{\mu}\right)^{2}-\frac{e}{2} m_{-}^{2}+i \lambda m_{+} \xi_{*}+i q \epsilon_{\mu \nu \lambda} \dot{x}^{\mu} \mathcal{A}^{\nu} \xi^{\lambda} \xi_{*}-\frac{i}{2} \xi \dot{\xi}-\frac{i}{2} \xi_{*} \dot{\xi}_{*}, \\
L_{B C L}^{g}=\frac{1}{2 e} \dot{x}^{2}-\frac{e}{2} m_{-}^{2}+\frac{i}{e m_{+}}\left(\dot{\xi}_{*}+q \epsilon_{\mu \nu \lambda} \dot{x}^{\mu} \mathcal{A}^{\nu} \xi^{\lambda}\right) \dot{x} \xi-\frac{i}{2} \xi_{\mu} \dot{\xi}^{\mu}+\frac{i}{2} \xi_{*} \dot{\xi}_{*},
\end{gathered}
$$

where $m_{ \pm}=m \pm \frac{i}{2} q \epsilon_{\mu \nu \lambda} \mathcal{A}^{\mu} \xi^{\nu} \xi^{\lambda}$. The comparison of the Lagrangians (7.1), (7.7) and (7.8) confirms the simplicity and naturalness of classical analog of $\mathrm{U}_{\sigma_{3}}(1)$ gauge theory for CPV model but its rather obscure and unnatural character for the case of BM and BCL models.

\section{Outlook}

We have observed the phenomenon of classical anomaly for three pseudoclassical models of $3 D P, T$-invariant system of planar fermions. One of the models is the CPV model having nilpotent constraint being nonlinear in Grassmann variables and admitting no, even local, gauge conditions. Because of this, the CPV model admits the quantization only by the Dirac method whereas the reduced phase space quantization cannot be applied to it. The application of path-integral method seems also to be problematic for such class of constrained systems [12. On the other hand, BM and BCL models contain linear in Grassmann variables nilpotent constraint and path-integral quantization method can in principle (see below) be applied for them. There are other pseudoclassical models containing nilpotent nonlinear constraints [15, [15]. Such models after quantization by the Dirac method describe the vector and higher spin fields. At least for one of such models, the model of $P, T$-invariant system of topologically massive vector U(1) gauge fields [16], some elements of classical anomaly were 
observed in Ref. [17]. Therefore, the natural question is whether it is possible to construct for any of peculiar higher spin models [15, 16] the 'supplementary' pseudoclassical model which would contain only linear nilpotent constraints but under quantization by the Dirac method would give the same quantum system as the corresponding known peculiar pseudoclassical model. If this question can be answered positively, the phenomenon of classical anomaly will be revealed for corresponding (equivalent quantum mechanically) pseudoclassical models.

In the same context it would be interesting to investigate different classical field theoretical models with odd fermions and also answer the intriguing question whether the phenomenon of classical anomaly can be observed for the models containing no Grassmann variables.

In conclusion we note that since the path-integral quantization method is closely related to the classical theory of the corresponding system to be quantized, it seems that the classical anomaly should reveal itself in some nontrivial way in path-integral approach to quantum mechanics. So, it seems to be very interesting to apply this method for quantizing the $3 D$ $\mathrm{BM}$ and BCL models.

\section{Acknowledgements}

J. G. was partially supported by grants 1950278 and 1960229 by FONDECYT-Chile and by grant 04-953/GR from Dicyt-USACH. M.P. thanks Prof. C. Teitelboim for discussions and University of Santiago of Chile, where the part of this work has been realized, for hospitality. One of us (J.G.) is a recipient of a John S. Guggenheim Fellowship.

\section{A Appendix}

Here we discuss the quantization of the BM and BCL models in $D=2 n$ case. In the BM model, the quantum counterparts of odd variables can be realized as

$$
\widehat{\xi}_{\mu}=\frac{1}{\sqrt{2}} \gamma_{*} \gamma_{\mu}, \quad \widehat{\xi}_{*}=\frac{1}{\sqrt{2}} \gamma_{*},
$$

where $\gamma_{\mu}$ are Dirac $2^{n} \times 2^{n}$-matrices satisfying the relations $\left[\gamma_{\mu}, \gamma_{\nu}\right]_{+}=-2 \eta_{\mu \nu}, \gamma_{\mu}^{\dagger}=-\eta_{\mu \mu} \gamma_{\mu}$ (no summation), whereas $\gamma_{*}=i^{n} \gamma_{0} \gamma_{1} \ldots \gamma_{d-1}, \gamma_{*}^{2}=-1, \gamma_{*}^{\dagger}=-\gamma_{*}$. As a result, the quantum analog of odd constraint coincides with the $2 n$-dimensional Dirac equation multiplied by nonsingular factor $\frac{1}{\sqrt{2}} \gamma_{*}$, and we arrive at the conclusion that the quantization of BM model in the case of $2 n$-dimensional space-time gives us the corresponding Dirac equation. The indefinite scalar product $\left\langle\Psi_{1} \mid \Psi_{2}\right\rangle=\bar{\Psi}_{1} \Psi_{2}$ with $\bar{\Psi}=\Psi^{\dagger} \Delta, \Delta=\sqrt{2} \widehat{\xi}^{0}$, guarantees that all the operators $\widehat{\xi}_{\mu}, \widehat{\xi}_{*}$, and as a consequence, all the observables are self-conjugate operators. The averaged odd constraint, $\langle\Psi \mid \widehat{\chi} \Psi\rangle=\Psi^{\dagger} \gamma^{0}(p \gamma+m) \Psi$, gives us the usual Lagrangian for $2 n$-dimensional massive Dirac fermion field.

The BCL model in $2 n$-dimensional space-time has $2 n+2$ odd phase space variables, i.e. one more in comparison with BM model. This difference is crucial for realization of quantum analogs of odd variables. The dimension of irreducible representation of the Clifford algebra with $2 n+2$ generators is $2^{n+1}$, twice more of that we had in BM model. As a result, quantum mechanically BM and BCL models are not equivalent in the case of $D=2 n$. Here one can realize odd operators in two different forms related by the corresponding unitary 
transformation. First, they can be realized as

$$
\widehat{\xi}_{\mu}=\frac{1}{\sqrt{2}} \gamma_{*} \gamma_{\mu} \otimes \sigma_{1}, \quad \widehat{\theta}_{1}=\frac{1}{\sqrt{2}} \gamma_{*} \otimes \sigma_{1}, \quad \widehat{\theta}_{0}=\frac{1}{\sqrt{2}} 1 \otimes \sigma_{3} .
$$

In this case the quantum analog of the odd constraint gives two $2 n$-dimensional Dirac equations (multiplied by nonsingular factor $\frac{1}{\sqrt{2}} \gamma_{*} \otimes \sigma_{1}$ ), and corresponding fermionic states are distinguished by the operator $\widehat{\theta}_{0}$. On the other hand, if we realize the Clifford algebra generators as

$$
\widehat{\xi}_{\mu}=\frac{1}{\sqrt{2}} \gamma_{\mu} \otimes \sigma_{1}, \quad \widehat{\theta}_{1}=\frac{i}{\sqrt{2}} 1 \otimes \sigma_{2}, \quad \widehat{\theta}_{0}=\frac{1}{\sqrt{2}} 1 \otimes \sigma_{3},
$$

the quantum counterpart of the odd constraint equation gives us the equation of the form (3.2). Realizations (A.2) and (A.3) are related by the unitary transformation $S \widehat{G} S^{-1}=\widehat{G}^{\prime}$, where $S=i\left(\gamma_{*} \otimes P_{+}-1 \otimes P_{-}\right), P_{ \pm}=\frac{1}{2}\left(1 \pm \sigma_{3}\right)$, and $\widehat{G}$ and $\widehat{G}^{\prime}$ are corresponding operators in realization (A.2) and (A.3), respectively.

Choosing the scalar product with indefinite metric operator $\Delta=\sqrt{2} \widehat{\xi}^{0}$, we get the operators $\widehat{\xi}_{\mu}$ and $\widehat{\theta}_{1}$ as self-conjugate ones. As a consequence, observables $\widehat{J}_{\mu \nu}$ and $\widehat{\Xi}_{\mu}$ will also be self-conjugate together with the quantum odd constraint which under averaging supplies us with the corresponding form of the Lagrangian, $\mathcal{L}=\Psi^{\dagger} \gamma^{0} \otimes 1(p \gamma \otimes 1+m \cdot 1 \otimes \Gamma) \Psi$, where $\Gamma=1$ and $\Gamma=\sigma_{3}$ for realizations (A.2) and (A.3). But as in the odd-dimensional case, the model again reveals the quantum anomaly: the quantum counterpart of the classical real observable variable $\theta_{0}$ turns out to be anti-self-conjugate.

Concluding, we note that in the case of $(3+1)$-dimensional BM model, three nontrivial transverse components $\widehat{\Xi}_{\mu}^{\perp}, \widehat{\Xi}_{\mu}^{\perp} p^{\mu}=0$, of the observable vector $\widehat{\Xi}_{\mu}$ are, in fact, the components of the Pauli-Lubanski vector for the Dirac field. On the other hand, the quantum BCL model gives us the pair of Dirac fields. In representation (A.2) the matrix part of the parity operator is $U_{P}=\gamma^{0} \otimes \sigma_{1}$. Therefore, the additional operator $\widehat{\theta}_{0}$ has here, as in the case of $2+1$ dimensions, the sense of parity-changing operator. As a result, the quantum BCL model, unlike the BM model, describes the pair of massive Dirac fermion fields having opposite internal parities.

\section{References}

[1] E. Witten, Phys. Lett. B 117 (1982) 324;

S. Elitzur, Y. Frishman, E. Rabinovici and A. Schwimmer, Nucl. Phys. B 273 (1986) 93 ;

J. Gamboa, V. O. Rivelles and J. Zanelli, Phys. Lett. B 395 (1997) 264.

[2] S. B. Treiman, R. Jackiw, B. Zumino and E. Witten, Currents, Algebras and Anomalies (World Scientific, Singapore, 1985).

[3] M. Henneaux and C. Teitelboim, Quantization of Gauge Systems (Princeton University Press, 1992).

[4] A. Kovner and B. Rosenstein, Phys. Rev. B 42 (1990) 4748;

G.W. Semenoff and N. Weiss, Phys. Lett. B 250 (1990) 117; 
N. Dorey and N.E. Mavromatos, Phys. Lett. B 250 (1990) 107; Nucl. Phys. B 386 (1992) 614.

[5] M. Plyushchay and P. Sodano, Hidden Supersymmetries of a P, T-invariant 3D Fermion System, Preprint hep-th/9511028.

[6] G. Grignani, M. Plyushchay and P. Sodano, Nucl. Phys. B 464 (1996) 189.

[7] F.A. Berezin and M.S. Marinov, JETP Letters 21 (1975) 678; Ann. Phys. 104 (1977) 336.

[8] L. Brink, S. Deser, B. Zumino, P. DiVecchia and P. Howe, Phys. Lett. B 64 (1976) 435;

L. Brink, P. DiVecchia and P.S. Howe, Nucl. Phys. B 118 (1977) 76.

[9] C.A. Galvao and C. Teitelboim, J. Math. Phys. 21 (1980) 1863.

[10] A. Barducci, R. Casalbuoni and L. Lusanna, Nuovo Cim. A 35 (1976) 377.

[11] J. L. Cortés, M. S. Plyushchay and L. Velázquez, Phys. Lett. B 306 (1993) 34.

[12] M. S. Plyushchay and A. V. Razumov, Int. J. Mod. Phys. A 11 (1996) 1427.

[13] K. Sundermeyer, Lecture Notes in Physics, Vol. 169 (Springer, Berlin, 1982).

[14] M. de Crombrugghe and V. Rittenberg, Ann. Phys. 151 (1983) 99.

[15] P.P. Srivastava, Nuovo Cim. Lett. 19 (1977) 239;

V.D. Gershun and V.I. Tkach, JETP Lett. 29 (1979) 320;

A. Barducci and L. Lusanna, J. Phys. A 16 (1983) 1993;

P.S. Howe, S. Penati, M. Pernici and P. Townsend, Phys. Lett. B 215 (1988) 555, Class. Quantum Grav. 6 (1989) 1125;

R. Marnelius and J. E. Martensson, Nucl. Phys. B 321 (1989) 185.

[16] Kh. S. Nirov and M. S. Plyushchay, Phys. Lett. B 405 (1997) 114.

[17] Kh. S. Nirov and M. S. Plyushchay, Nucl. Phys. B 512 (1998) 295. 